\title{
Simulating Dynamical Features of Escape Panic
}

\author{
Dirk Helbing ${ }^{*,+}$, Illés Farkas,$^{\ddagger}$ and Tamás Vicsek ${ }^{*, \ddagger}$ \\ * Collegium Budapest - Institute for Advanced Study, Szentháromság u. 2, \\ H-1014 Budapest, Hungary \\ + Institute for Economics and Traffic, Dresden University of Technology, \\ D-01062 Dresden, Germany \\ $\ddagger$ Department of Biological Physics, Eötvös University, \\ Pázmány Péter Sétány 1A, H-1117 Budapest, Hungary \\ helbing@trafficforum.de; fij@elte.hu; vicsek@angel.elte.hu
}

One of the most disastrous forms of collective human behaviour is the kind of crowd stampede induced by panic, often leading to fatalities as people are crushed or trampled. Sometimes this behaviour is triggered in life-threatening situations such as fires in crowded buildings $;^{1,2}$ at other times, stampedes can arise from the rush for seats $^{3,4}$ or seemingly without causes. Tragic examples within recent months include the panics in Harare, Zimbabwe, and at the Roskilde rock concert in Denmark. Although engineers are finding ways to alleviate the scale of such disasters, their frequency seems to be increasing with the number and size of mass events. ${ }^{2,5}$ Yet, systematic studies of panic behaviour ${ }^{6-9}$ and quantitative theories capable of predicting such crowd dynamics, ${ }^{5,10-12}$ are rare. Here we show that simulations based on a model of pedestrian behaviour can provide valuable insights into the mechanisms of and preconditions for panic and jamming by incoordination. Our results suggest practical ways of minimising the harmful consequences of such events 


\section{and the existence of an optimal escape strategy, corresponding to a suitable mixture of individualistic and collective behaviour.}

Up to now, panics as a particular form of collective behaviour occuring in situations of scarce or dwindling resources ${ }^{1,6}$ has been mainly studied from the perspective of social psychology. ${ }^{7-9}$ Panicking individuals tend to show maladaptive and relentless mass behaviour like jamming and life-threatening overcrowding, ${ }^{1-4,8}$ which has often been attributed to social contagion ${ }^{1,4,8}$ (see Brown ${ }^{9}$ for an overview of theories). According to Mintz, ${ }^{6}$ the observed jamming is a result of incoordination and depends on the reward structure.

After having carefully studied the related socio-psychological literature ${ }^{6-9}$ reports in the media and available video materials (see http://angel.elte. $\mathrm{hu} / \sim$ panic/), empirical investigations,,${ }^{1,2,3}$ and engineering handbooks, ${ }^{13,14}$ we can summarise the following characteristic features of escape panics: (i) People move or try to move considerably faster than normal. ${ }^{13}$ (ii) Individuals start pushing, and interactions among people become physical in nature. (iii) Moving and, in particular, passing of a bottleneck becomes incoordinated. ${ }^{6}$ (iv) At exits, arching and clogging are observed. ${ }^{13}$ (v) Jams are building up. ${ }^{7}$ (vi) The physical interactions in the jammed crowd add up and cause dangerous pressures up to 4,450 Newtons per meter, ${ }^{2,5}$ which can bend steel barriers or tear down brick walls. (vii) Escape is further slowed down by fallen or injured people turning into "obstacles". (viii) People show a tendency of mass behaviour, i.e., to do what other people do. ${ }^{1,8}$ (ix) Alternative exits are often overlooked or not efficiently used in escape situations. ${ }^{1,2}$

These observations have encouraged us to model the collective phenomenon of escape panic in the spirit of self-driven many-particle systems. Our computer 
simulations of the crowd dynamics of pedestrians are based on a generalised force model, ${ }^{15}$ which is particularly suited to describe the fatal build up of pressure observed during panics. ${ }^{2-4,5}$ We assume a mixture of socio-psychological ${ }^{16}$ and physical forces influencing the behaviour in a crowd: Each of $N$ pedestrians $i$ of mass $m_{i}$ likes to move with a certain desired speed $v_{i}^{0}$ into a certain direction $\boldsymbol{e}_{i}^{0}$, and therefore tends to correspondingly adapt his or her actual velocity $\boldsymbol{v}_{i}$ with a certain characteristic time $\tau_{i}$. Simultaneously, he or she tries to keep a velocity-dependent distance to other pedestrians $j$ and walls $W$. This can be modelled by "interaction forces" $\boldsymbol{f}_{i j}$ and $\boldsymbol{f}_{i W}$, respectively. In mathematical terms, the change of velocity in time $t$ is then given by the acceleration equation

$$
m_{i} \frac{d \boldsymbol{v}_{i}}{d t}=m_{i} \frac{v_{i}^{0}(t) \boldsymbol{e}_{i}^{0}(t)-\boldsymbol{v}_{i}(t)}{\tau_{i}}+\sum_{j(\neq i)} \boldsymbol{f}_{i j}+\sum_{W} \boldsymbol{f}_{i W},
$$

while the change of position $\boldsymbol{r}_{i}(t)$ is given by the velocity $\boldsymbol{v}_{i}(t)=d \boldsymbol{r}_{i} / d t$. We describe the psychological tendency of two pedestrians $i$ and $j$ to stay away from each other by a repulsive interaction force $A_{i} \exp \left[\left(r_{i j}-d_{i j}\right) / B_{i}\right] \boldsymbol{n}_{i j}$, where $A_{i}$ and $B_{i}$ are constants. $d_{i j}=\left\|\boldsymbol{r}_{i}-\boldsymbol{r}_{j}\right\|$ denotes the distance between the pedestrians' centers of mass, and $\boldsymbol{n}_{i j}=\left(n_{i j}^{1}, n_{i j}^{2}\right)=\left(\boldsymbol{r}_{i}-\boldsymbol{r}_{j}\right) / d_{i j}$ is the normalised vector pointing from pedestrian $j$ to $i$. If their distance $d_{i j}$ is smaller than the sum $r_{i j}=\left(r_{i}+r_{j}\right)$ of their radii $r_{i}$ and $r_{j}$, the pedestrians touch each other. In this case, we assume two additional forces inspired by granular interactions, ${ }^{17,18}$ which are essential for understanding the particular effects in panicking crowds: a "body force" $k\left(r_{i j}-d_{i j}\right) \boldsymbol{n}_{i j}$ counteracting body compression and a "sliding friction force" $\kappa\left(r_{i j}-d_{i j}\right) \Delta v_{j i}^{t} \boldsymbol{t}_{i j}$ impeding relative tangential motion, if pedestrian $i$ comes close to $j$. Herein, $\boldsymbol{t}_{i j}=\left(-n_{i j}^{2}, n_{i j}^{1}\right)$ means the tangential direction and $\Delta v_{j i}^{t}=\left(\boldsymbol{v}_{j}-\boldsymbol{v}_{i}\right) \cdot \boldsymbol{t}_{i j}$ the tangential velocity 
difference, while $k$ and $\kappa$ represent large constants. In summary, we have

$$
\boldsymbol{f}_{i j}=\left\{A_{i} \exp \left[\left(r_{i j}-d_{i j}\right) / B_{i}\right]+k g\left(r_{i j}-d_{i j}\right)\right\} \boldsymbol{n}_{i j}+\kappa g\left(r_{i j}-d_{i j}\right) \Delta v_{j i}^{t} \boldsymbol{t}_{i j},
$$

where the function $g(x)$ is zero, if the pedestrians do not touch each other $\left(d_{i j}>r_{i j}\right)$, otherwise equal to the argument $x$.

The interaction with the walls is treated analogously, i.e., if $d_{i W}$ means the distance to wall $W, \boldsymbol{n}_{i W}$ denotes the direction perpendicular to it, and $\boldsymbol{t}_{i W}$ the direction tangential to it, the corresponding interaction force with the wall reads

$\boldsymbol{f}_{i W}=\left\{A_{i} \exp \left[\left(r_{i}-d_{i W}\right) / B_{i}\right]+k g\left(r_{i}-d_{i W}\right)\right\} \boldsymbol{n}_{i W}-\kappa g\left(r_{i}-d_{i W}\right)\left(\boldsymbol{v}_{i} \cdot \boldsymbol{t}_{i W}\right) \boldsymbol{t}_{i W}$.

Probably due to the fact that escape panics are unexpected and dangerous events, which also excludes real-life experiments, we could not find suitable data on escape panics to test our model quantitatively. This scarcity of data calls for reliable models. We have, therefore, specified the parameters as follows: With a mass of $m_{i}=80 \mathrm{~kg}$, we represent an average soccer fan. The desired velocity $v_{i}^{0}$ can reach more than $5 \mathrm{~m} / \mathrm{s}$ (up to $10 \mathrm{~m} / \mathrm{s}$ ), ${ }^{14}$ but the observed free velocities for leaving a room correspond to $v_{i}^{0} \approx 0.6 \mathrm{~m} / \mathrm{s}$ under relaxed, $v_{i}^{0} \approx 1 \mathrm{~m} / \mathrm{s}$ under normal, and $v_{i}^{0} \lesssim 1.5 \mathrm{~m} / \mathrm{s}$ under nervous conditions. ${ }^{13}$ A reasonable estimate for the acceleration time is $\tau_{i}=0.5 \mathrm{~s}$. With $A_{i}=2 \cdot 10^{3} \mathrm{~N}$ and $B_{i}=0.08 \mathrm{~m}$ one can reflect the distance kept at normal desired velocities ${ }^{14}$ and fit the measured flows through bottlenecks ${ }^{14}$, amounting to 0.73 persons per second for an effectively 1 meter wide door under conditions with $v_{i}^{0} \approx 0.8 \mathrm{~m} / \mathrm{s}$. The parameters $k=1.2 \cdot 10^{5} \mathrm{~kg} \mathrm{~s}^{-2}$ and 
$\kappa=2.4 \cdot 10^{5} \mathrm{~kg} \mathrm{~m}^{-1} \mathrm{~s}^{-1}$ determine the obstruction effects in cases of physical interactions. Although, in reality, most parameters are varying individually, we chose identical values for all pedestrians to minimise the number of parameters for reasons of calibration and robustness, and to exclude irregular outflows because of parameter variations. However, to avoid model artefacts (gridlocks by exactly balanced forces in symmetrical configurations), a small amount of irregularity of almost arbitrary kind is needed. This irregularity was introduced by uniformly distributed pedestrian diameters $2 r_{i}$ in the interval $[0.5 \mathrm{~m}, 0.7 \mathrm{~m}]$, approximating the distribution of shoulder widths of soccer fans.

Based on the above model assumptions, we will now simulate several important phenomena of escape panic, which are insensitive to reasonable parameter variations, but fortunately become less pronounced for wider exits.

1. Transition to Incoordination due to Clogging. The simulated outflow from a room is well-coordinated and regular, if the desired velocities $v_{i}^{0}=v_{0}$ are normal. However, for desired velocities above $1.5 \mathrm{~m} / \mathrm{s}$, i.e. for people in a rush, we find an irregular succession of arch-like blockings of the exit and avalanchelike bunches of leaving pedestrians, when the arches break (see Fig. 1a, b). This phenomenon is compatible with the empirical observations mentioned above and comparable to intermittent clogging found in granular flows through funnels or hoppers ${ }^{17,18}$ (although this has been attributed to static friction between particles without remote interactions, and the transition to clogging has been observed for small enough openings rather than for a variation of the driving force).

2. "Faster-Is-Slower Effect" due to Impatience. Since clogging is connected 
with delays, trying to move faster (i.e., increasing $v_{i}^{0}$ ) can cause a smaller average speed of leaving, if the friction parameter $\kappa$ is large enough (see Fig. 1c, d). This "faster-is-slower effect" is particularly tragic in the presence of fires, where fleeing people reduce their own chances of survival. The related fatalities can be estimated by the number of pedestrians reached by the fire front (see http://angel.elte.hu/ ${ }^{\sim}$ panic/).

Since our friction term has, on average, no deceleration effect in the crowd, if the walls are sufficiently remote, the arching underlying the clogging effect requires a combination of several effects: i) slowing down due to a bottleneck such as a door and ii) strong inter-personal friction, which becomes dominant when pedestrians get too close to each other. Consequently, the danger of clogging can be minimised by avoiding bottlenecks in the construction of stadia and public buildings. Notice, however, that jamming can also occur at widenings of escape routes! This surprising result is illustrated in Fig. 2. Improved outflows can be reached by columns placed asymmetrically in front of the exits, which also prevent the build up of fatal pressures (see http://angel.elte.hu/ panic/).

3. Mass Behaviour. We investigate a situation in which pedestrians are trying to leave a smoky room, but first have to find one of the invisible exits (see Fig. 3a). Each pedestrian $i$ may either select an individual direction $\boldsymbol{e}_{i}$ or follow the average direction $\left\langle\boldsymbol{e}_{j}^{0}(t)\right\rangle_{i}$ of his neighbours $j$ in a certain radius $R_{i}{ }^{19}$ or try a mixture of both. We assume that both options are weighted with some parameter $p_{i}$ :

$$
\boldsymbol{e}_{i}^{0}(t)=\mathcal{N}\left[\left(1-p_{i}\right) \boldsymbol{e}_{i}+p_{i}\left\langle\boldsymbol{e}_{j}^{0}(t)\right\rangle_{i}\right]
$$


where $\mathcal{N}(\boldsymbol{z})=\boldsymbol{z} /\|\boldsymbol{z}\|$ denotes normalisation of a vector $\boldsymbol{z}$. As a consequence, we have individualistic behaviour if $p_{i}$ is low, but herding behaviour if $p_{i}$ is high. Therefore, $p_{i}$ reflects the degree of panics of individual $i$.

Our model suggests that neither individualistic nor herding behaviour performs well (see Fig. 3b). Pure individualistic behaviour means that each pedestrian finds an exit only accidentally, while pure herding behaviour implies that the complete crowd is eventually moving into the same and probably blocked direction, so that available exits are not efficiently used, in agreement with observations. According to Figs. 3b and c, we expect optimal chances of survival for a certain mixture of individualistic and herding behaviour, where individualism allows some people to detect the exits and herding guarantees that successful solutions are imitated by the others. If pedestrians follow the walls instead of "reflecting" at them, we expect that herd following causes jamming and inefficient use of doors as well (see Fig. 1), while individualists moving in opposite directions obstruct each other.

In summary, we have developed a continuous pedestrian model based on plausible interactions, which is, due to its simplicity, robust with respect to parameter variations and suitable for drawing conclusions about the possible mechanisms beyond escape panic (regarding an increase of the desired velocity, strong friction effects during physical interactions, and herding). After having calibrated the model parameters to available data on pedestrian flows, we managed to reproduce many observed phenomena including i) the build up of pressure, ii) clogging effects at bottlenecks, iii) jamming at widenings, iv) the "faster-is-slower effect", v) inefficient use of alternative exits (see Fig. 3d), and vi) initiation of panics by counterflows and impatience (i.e., seemingly 
without any logical reason, see Fig. 1). Moreover, we are able to simulate situations of dwindling resources and estimate the casualties. Therefore, the model can be used to test buildings for their suitability in emergency situations. It accounts for both, the different dynamics in normal and panic situations just by changing a single parameter $p_{i}=p$. In addition, our simulations suggest that the optimal behaviour in escape situations is a suitable mixture of individualistic and herding behaviour.

We are now calling for complementary data and additional video material on escape panics to test our model quantitatively and compare it with alternative ones which, for example, include direction- and velocity-dependent interpersonal interactions, specify the individual variation of parameters, study the effect of fluctuations, consider falling people, integrate acoustic information exchange, implement more complex strategies and interactions (also threedimensional ones), or allow for switching of strategies. A superior theory would have to reproduce the empirical findings equally well with less parameters, reach a better quantitative agreement with data with the same number, or reflect additional observations.

\section{REFERENCES}

1. Keating, J. P. The myth of panic. Fire Journal, 57-61+147 (May/1982).

2. Elliott, D. \& Smith, D. Football stadia disasters in the United Kingdom: learning from tragedy? Industrial \&6 Environmental Crisis Quarterly 7(3), 205-229 (1993).

3. Jacobs, B. D. \& 't Hart, P. Disaster at Hillsborough Stadium: a comparative analysis. Chap. 10 in Parker, D. J. \& Handmer, J. W. Hazard 
Helbing/Farkas/Vicsek: Simulating Dynamical Features of Escape Panic 9 Management and Emergency Planning (James \& James Science, London, 1992).

4. Johnson, N. R. Panic at "The Who Concert Stampede": an empirical assessment. Social Problems 34(4), 362-373 (1987).

5. Smith, R. A. \& Dickie, J. F. (eds.) Engineering for Crowd Safety (Elsevier, Amsterdam, 1993).

6. Mintz, A. Non-adaptive group behavior. The Journal of Abnormal and Normal Social Psychology 46, 150-159 (1951).

7. Kelley, H. H, Condry, J. C. Jr., Dahlke, A. E \& Hill, A. H. Collective behavior in a simulated panic situation. Journal of Experimental Social Psychology 1, 20-54 (1965).

8. Quarantelli, E. The behavior of panic participants. Sociology and Social Research 41, 187-194 (1957).

9. Brown, R. Social Psychology (The Free Press, New York, 1965).

10. Drager, K. H., Løvås, G., Wiklund, J., Soma, H., Duong, D., Violas, A. \& Lanèrès, V. EVACSIM - A comprehensive evacuation simulation tool, in the Proceedings of the 1992 Emergency Management and Engineering Conference (Society for Computer Simulation, Orlando, Florida, 1992), pp. 101-108.

11. Ebihara, M., Ohtsuki, A. \& Iwaki, H. A model for simulating human behavior during emergency evacuation based on classificatory reasoning and certainty value handling. Microcomputers in Civil Engineering 7, 63-71 (1992).

12. Still, G. K. New computer system can predict human behaviour response to building fires. Fire 84, 40-41 (1993).

13. Predtetschenski, W. M. \& Milinski, A. I. Personenströme in Gebäuden 
Helbing/Farkas/Vicsek: Simulating Dynamical Features of Escape Panic 10

- Berechnungsmethoden für die Projektierung - (Rudolf Müller, KölnBraunsfeld, 1971).

14. Weidmann, U. Transporttechnik der Fußgänger (Institut für Verkehrsplanung, Transporttechnik, Straßen- und Eisenbahnbau (IVT), ETH Zürich, 1993).

15. Helbing, D., Farkas, I. J. \& Vicsek, T. Freezing by heating in a driven mesoscopic system. Physical Review Letters 84, 1240-1243 (2000).

16. Helbing, D. \& Molnár, P. Social force model for pedestrian dynamics. Physical Review E 51, 4282-4286 (1995).

17. Ristow, G. H. \& Herrmann, H. J. Density patterns in two-dimensional hoppers. Physical Review E 50, R5-R8 (1994).

18. Wolf, D. E. \& Grassberger, P. (eds.) Friction, Arching, Contact Dynamics (World Scientific, Singapore, 1997).

19. Vicsek, T., Czirók, A., Ben-Jacob, E., Cohen, I. \& Shochet, O. Novel type of phase transition in a system of self-driven particles. Physical Review Letters 75, 1226-1229 (1995).

20. Kelley, H. H. \& Thibaut, J. W. Group problem solving. In Lindzey, G. \& Aronson, E. (eds.) The Handbook of Social Psychology, Vol. 4 (Addison-Wesley, Reading, MA, 1969).

Acknowledgments: D.H. thanks the German Research Foundation (DFG) for financial support by a Heisenberg scholarship. T.V. and I.F. are grateful for partial support by OTKA and FKFP. 
Helbing/Farkas/Vicsek: Simulating Dynamical Features of Escape Panic 11

\section{FIGURES}

a
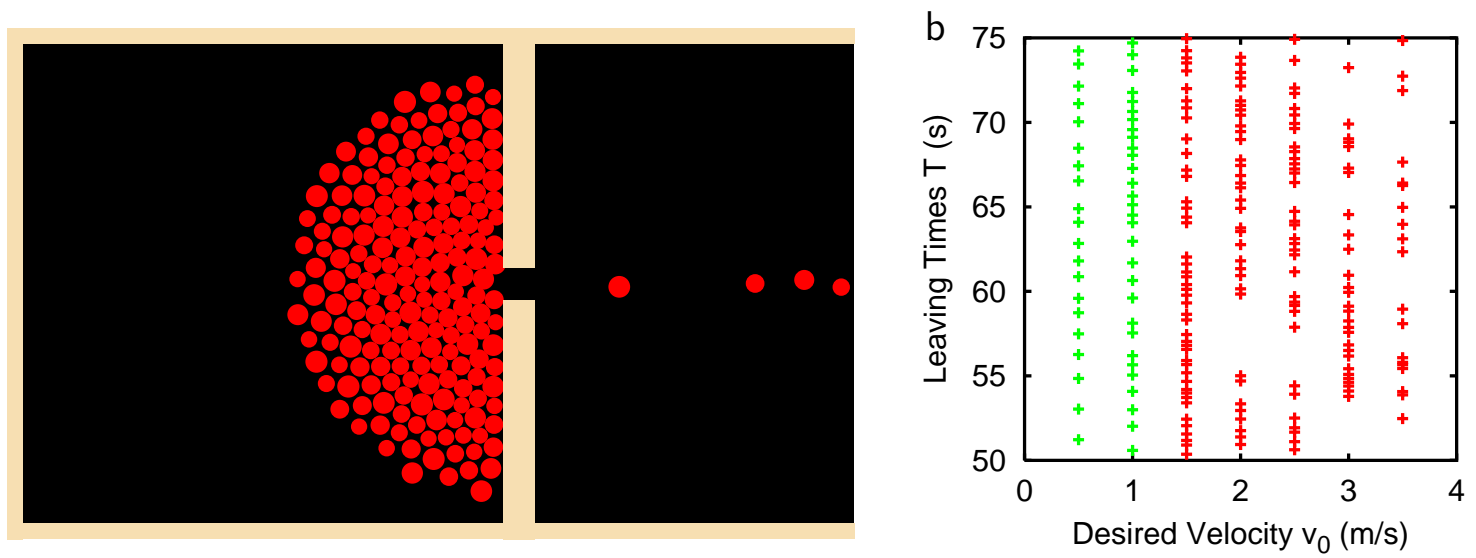

C

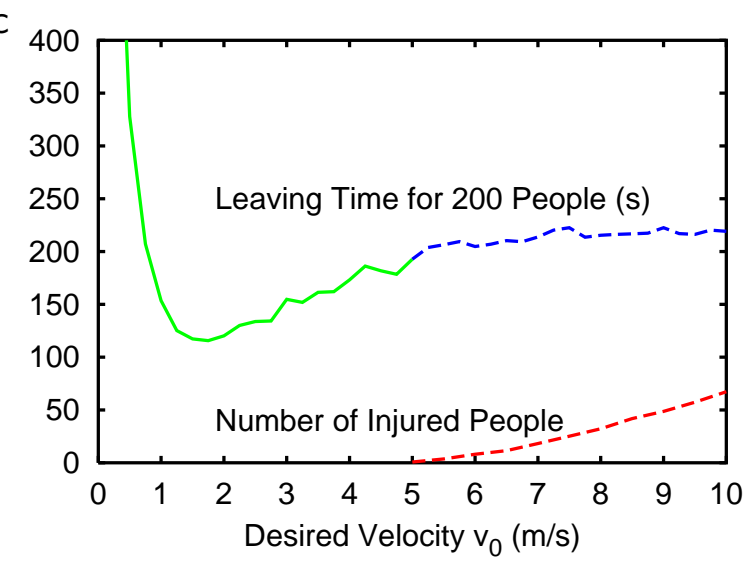

$d_{1.4}$

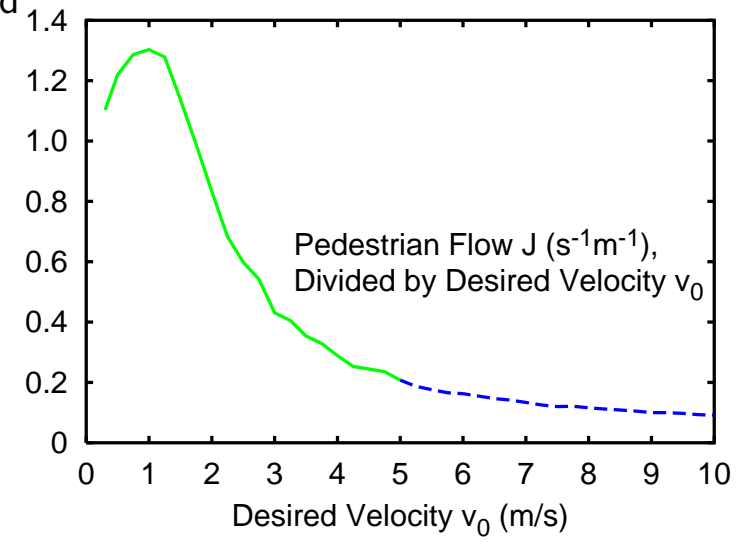


FIG. 1. Simulation of pedestrians moving with identical desired velocity $v_{i}^{0}=v_{0}$ towards the $1 \mathrm{~m}$ wide exit of a room of size $15 \mathrm{~m} \times 15 \mathrm{~m}$. a Snapshot of the scenario. Dynamic simulations are available at http://angel.elte.hu/ panic/. b Illustration of leaving times of pedestrians for various desired velocities $v_{0}$. Irregular outflow due to clogging is observed for high desired velocities $\left(v_{0} \geq 1.5 \mathrm{~m} / \mathrm{s}\right.$, see red plusses). c Under conditions of normal walking, the time for 200 pedestrians to leave the room decreases with growing $v_{0}$. Desired velocities higher than $1.5 \mathrm{~m} / \mathrm{s}$ reduce the efficiency of leaving, which becomes particularly clear, when the outflow $J$ is divided by the desired velocity (see d). This is due to pushing, which causes additional friction effects. Moreover, above a desired velocity of about $v_{0}=5 \mathrm{~m} / \mathrm{s}$ $(--)$, people are injured and become non-moving obstacles for others, if the sum of the magnitudes of the radial forces acting on them divided by their circumference exceeds a pressure of $1600 \mathrm{~N} / \mathrm{m} .^{5}$

Due to the above "faster-is-slower effect", panics can be triggered by pedestrian counterflows, ${ }^{2}$ which cause delays to the crowd intending to leave. This makes the stopped pedestrians impatient and pushy. One may describe this by increasing the desired velocity according to $v_{i}^{0}(t)=\left[1-p_{i}(t)\right] v_{i}^{0}(0)+p_{i}(t) v_{i}^{\max }$, where $v_{i}^{0}(0)$ is the initial and $v_{i}^{\max }$ the maximum desired velocity. The time-dependent parameter $p_{i}(t)=1-\bar{v}_{i}(t) / v_{i}^{0}$, where $\bar{v}_{i}(t)$ denotes the average speed into the desired direction of motion, is a measure of impatience. Altogether, long waiting times increase the desired velocity, which can produce inefficient outflow. This further increases the waiting times, and so on, so that this tragic feedback can eventually trigger panics. It is, therefore, imperative, to have sufficiently wide exits and to prevent counterflows, when big crowds want to leave. 
Helbing/Farkas/Vicsek: Simulating Dynamical Features of Escape Panic 13
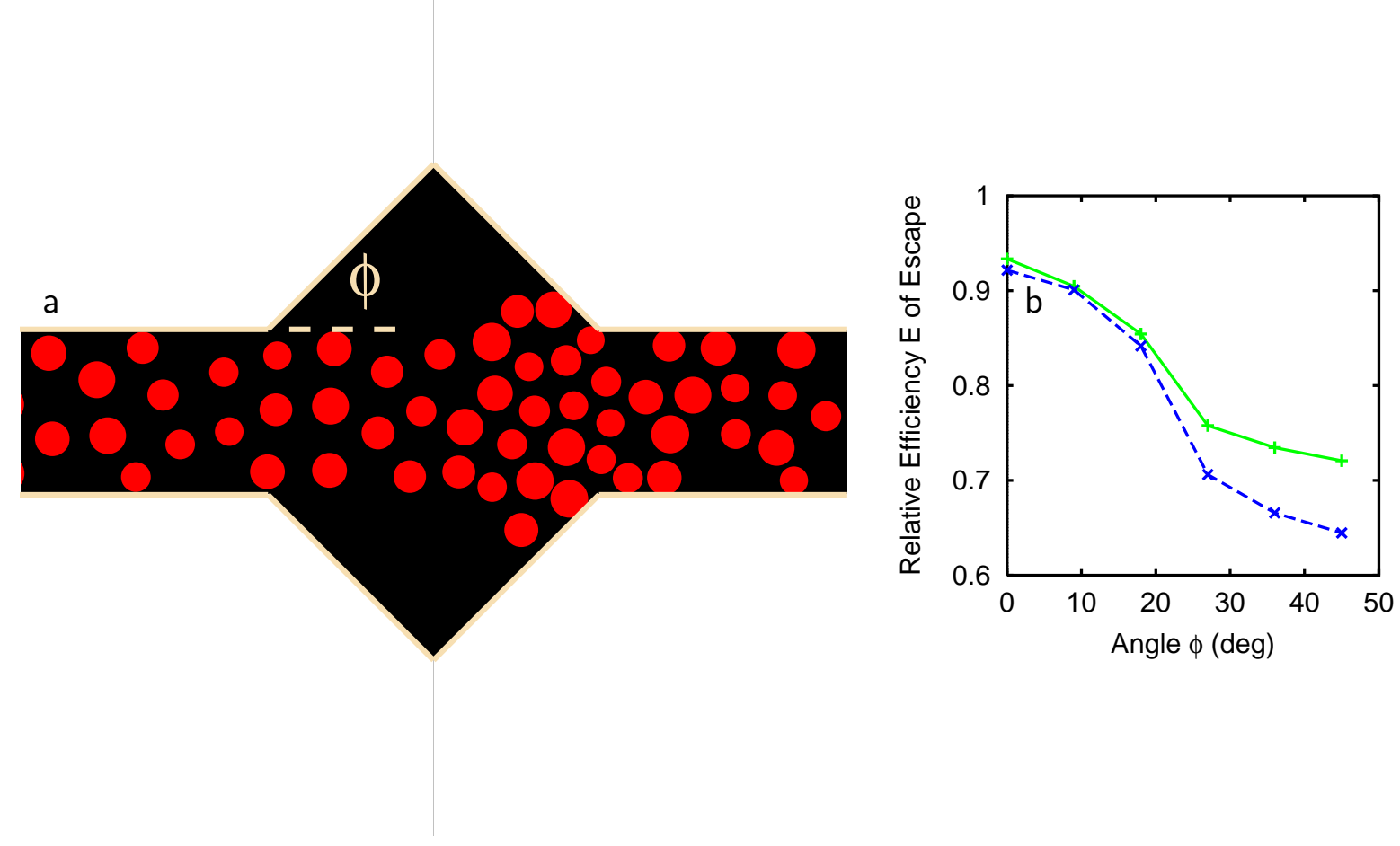
FIG. 2. Simulation of an escape route with a wider area (see also the Java applets supplied at http://angel.elte.hu/ panic/). a Illustration of the scenario with $v_{i}^{0}=v_{0}=2 \mathrm{~m} / \mathrm{s}$. The corridor is $3 \mathrm{~m}$ wide and $15 \mathrm{~m} \mathrm{long}$, the length of the triangular pieces in the middle being $2 \times 3 \mathrm{~m}=6 \mathrm{~m}$. Pedestrians enter the simulation area on the left-hand side with an inflow of $J=5.5 \mathrm{~s}^{-1} \mathrm{~m}^{-1}$ and flee towards the right-hand side. b Efficiency of leaving as a function of the angle $\phi$ characterising the width of the central zone, i.e., the difference from a linear corridor. The relative efficiency $E=\left\langle\boldsymbol{v}_{i} \cdot \boldsymbol{e}_{i}^{0}\right\rangle / v_{0}$ measures the average velocity along the corridor compared to the desired velocity and lies between 0 and $1(-)$. While it is almost one (i.e., maximal) for a linear corridor $(\phi=0)$, the efficiency drops by about $20 \%$, if the corridor contains a widening. This becomes comprehensible, if we take into account that the widening leads to disturbances by pedestrians, who expand in the wide area due to their repulsive interactions or try to overtake each other, and squeeze into the main stream again at the end of the widening. Hence, the right half of the illustrated corridor acts like a bottleneck and leads to jamming. The drop of efficiency $E$ is even more pronounced, (i) in the area of the widening where pedestrian flow is most irregular (--), (ii) if the corridor is narrow, (iii) if the pedestrians have different or high desired velocities, and (iv) if the pedestrian density in the corridor is higher. 
a
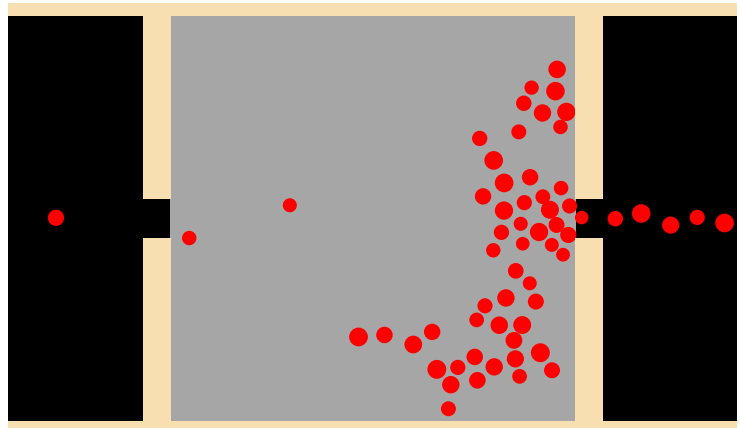

C

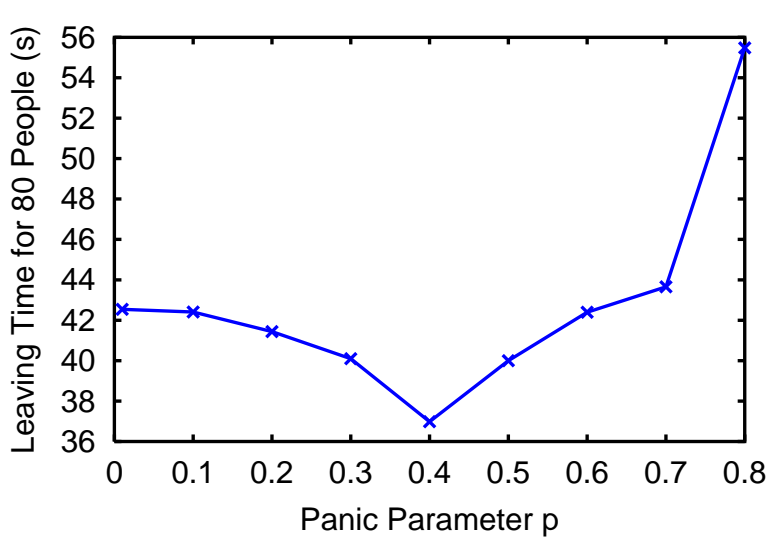

b
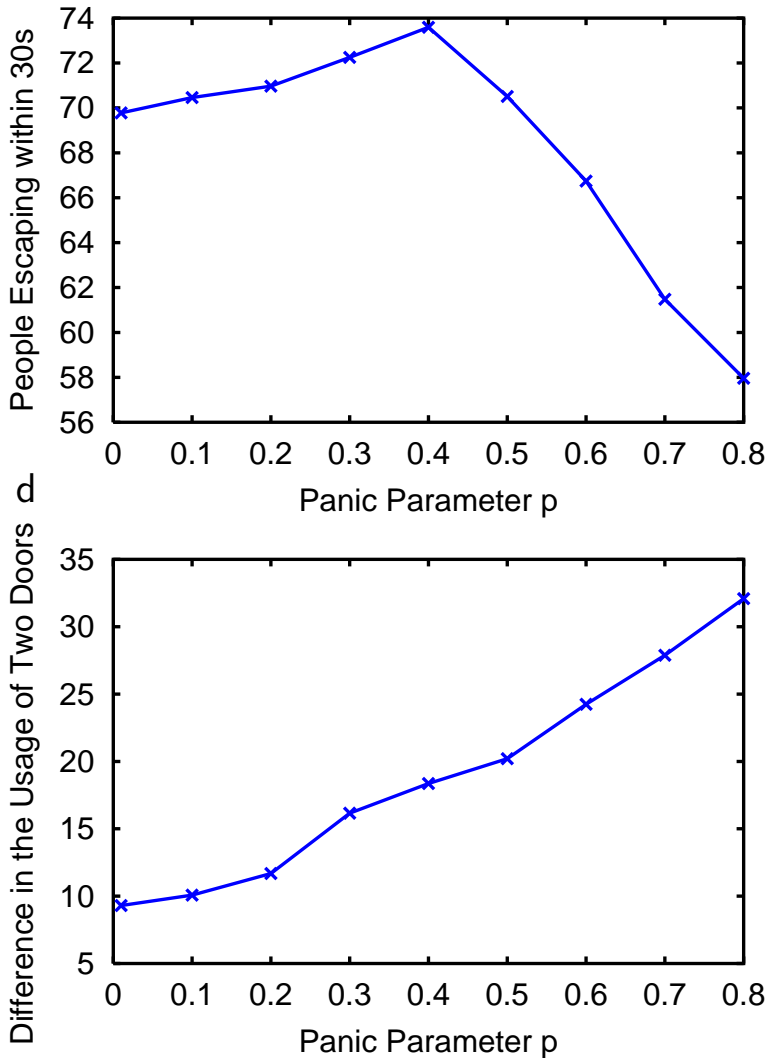
FIG. 3. Simulation of $N=90$ pedestrians trying to escape a smoky room of area $A=15 \mathrm{~m} \times 15 \mathrm{~m}$ (grey) through two invisible doors of $1.5 \mathrm{~m}$ width, which have to be found with a mixture of individualistic and herding behaviour. Java applets are available at http://angel.elte.hu/ panic/. a Snapshot of the simulation with $v_{i}^{0}=v_{0}=5 \mathrm{~m} / \mathrm{s}$. Initially, each pedestrian selects his or her desired walking direction randomly. Afterwards, a pedestrian's walking direction is influenced by the average direction of the neighbours within a radius of, for example, $R_{i}=R=5 \mathrm{~m}$. The strength of this herding effect grows with increasing panic parameter $p_{i}=p$ and increasing value of $h=\pi R^{2} \rho$, where $\rho=N / A$ denotes the pedestrian density. When reaching a boundary, the direction of a pedestrian is reflected. If one of the exits is closer than $2 \mathrm{~m}$, the room is left. $\mathrm{b}$ Number of people who manage to escape within $30 \mathrm{~s}$ as a function of the panic parameter $p$. c Illustration of the time required by 80 individuals to leave the smoky room. If the exits are relatively narrow and the degree $p$ of herding is small or large, leaving takes particularly long, so that only some of the people escape before being poisoned by smoke. Our results suggest that the best escape strategy is a certain compromise between following of others and an individualistic searching behaviour. This fits well into experimental data on the efficiency of group problem solving, ${ }^{20}$ according to which groups normally perform better than individuals, but masses are inefficient in finding new solutions to complex problems. d Absolute difference $\left|N_{1}-N_{2}\right|$ in the numbers $N_{1}$ and $N_{2}$ of persons leaving through the left exit or the right exit as a function of the herding parameter $p$. We find that pedestrians tend to jam up at one of the exits instead of equally using all available exits, if the panic parameter is large. 